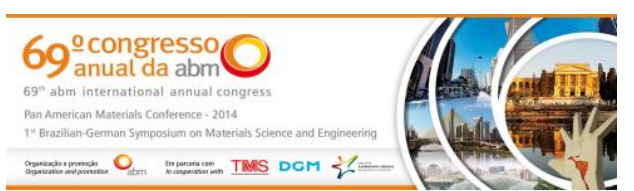

Tema: Diagramas de fases e transformações de fases

\title{
SIMULAÇÃO DE REAÇÕES COM NUCLEAÇÃO E CRESCIMENTO DE FASES SEQUENCIAIS PELO MÉTODO DO CONE CAUSAL*
}

\author{
André Luiz Moraes Alves ${ }^{1}$ \\ Weslley Luiz da Silva Assis² \\ Simone Carreiro de Oliveira ${ }^{3}$ \\ Paulo Rangel Rios ${ }^{4}$
}

\section{Resumo}

As transformações sequenciais estão presentes em vários materiais, como por exemplo, nos aços, onde na transformação da Austenita em Ferrita e Perlita, tem-se inicialmente a nucleação dos grãos ferríticos e em um instante posterior a nucleação dos grãos perlíticos. Recentemente, Rios e Villa apresentaram um método analítico exato que se aplica às transformações de fase simultâneas e/ou sequenciais. Neste trabalho o método do Cone Causal (CC) foi utilizado para o modelamento microestrutural das transformações sequenciais. Vale ressaltar que o CC é um método computacional muito versátil, sua eficiência em simulações de nucleação e crescimento já foi apresentada em trabalhos prévios. Os dados obtidos nas simulações foram comparados com as equações analíticas de Rios e Villa. A simulação permite obter, além da microestrutura propriamente dita, grandezas microestruturais quantitativas, tais como: fração volumétrica transformada, área superficial por unidade de volume, velocidade de avanço das interfaces e o caminho microestrutural.

Palavras-chave: Recristalização; Reações sequenciais; Simulação Computacional.

\section{SIMULATION OF REACTION WITH NUCLEATION AND GROWTH OF SEQUENTIAL PHASES BY THE CAUSAL CONE METHOD}

\section{Abstract}

Sequential transformations are present in various materials, for example in steel, where the transformation of Austenite into Ferrite and Perlite has first nucleation of ferritic grains and at a later instant perlitic grains. Recently, Rios and Villa presented an exact analytical method that applies to simultaneous and/or sequential transformations. In this work the method of Causal Cone (CC) was used for the microstructural modeling of sequential transformations. The $\mathrm{CC}$ is a very versatile computational method, its efficiency in simulations of nucleation and growth was already presented. The data obtained in the simulations were compared with the analytical equations of Rios and Villa. The simulation allows to obtain, besides the microstructure, quantitative microstructural quantities such as the volume fraction transformed, surface area per unit volume, forward speed interfaces and microstructural path.

Keywords: Recrystallization; Sequential reactions; Computer simulation.

\footnotetext{
Eng. Metalúrgico, Mestrando em Engenharia Metalúrgica, UFF, Volta Redonda, RJ, Brasil. Eng. Químico, Pós-Doutorando em Engenharia Metalúrgica, UFF, Volta Redonda, RJ, Brasil. Eng. Metalúrgica, Doutoranda em Engenharia Metalúrgica, UFF, Volta Redonda, RJ, Brasil. Prof. PhD em Engenharia Metalúrgica, Prof. Titular do curso de pós-graduação em Engenharia Metalúrgica, UFF, Volta Redonda, RJ, Brasil.
}

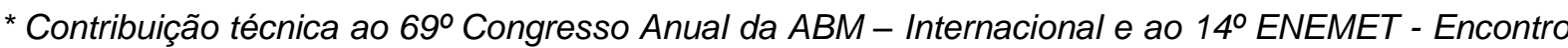
Nacional de Estudantes de Engenharia Metalúrgica, de Materiais e de Minas, 21 a 25 de julho de 2014, São Paulo, SP, Brasil.
} 


\section{INTRODUÇÃO}

A metodologia da cinética formal é frequentemente empregada para analisar uma variedade de transformações heterogêneas em fases condensadas [1-3]. Esta metodologia teve início com os trabalhos de Johnson [4], Mehl [5], Kolmogorov [6] e Avrami [7,8].

A recristalização é um fenômeno microestrutural bem conhecido. Ela é objeto de muitos estudos e ocorre a partir de uma matriz que sofreu deformação prévia. De uma maneira generalizada pode ser estudada em função de duas etapas básicas, nucleação e crescimento.

Rios e Villa [9] desenvolveram um método analítico novo e matematicamente exato para modelar situações em que mais de uma transformação ocorre simultaneamente e/ou sequencialmente. Este novo método permite se construir modelos analíticos para duas ou mais reações a partir dos modelos individuais teóricos. Além disto, permite extrair a cinética das reações individuais a partir da cinética total de transformação. O método analítico, entretanto, fornece apenas valores de parâmetros estereológicos, tais como: fração volumétrica, área por unidade de volume e caminho microestrutural.

Neste trabalho utilizou-se o método do Cone Causal para simular reações sequenciais. A partir dos resultados gerados pela simulação computacional foi possível comparar a solução analítica com a simulação. Além da construção dos gráficos geraram-se as microestruturas finais e intermediárias.

Um exemplo de aplicação da metodologia foi descrita no trabalho de Rios, Villa e Oliveira [10], no qual foi utilizado o novo modelo analítico para analisar dados publicados por Magnusson, Jensen e Hutchinsson [11], sobre a cinética de recristalização de aços IF. A partir dessa análise, provou-se que o método é eficiente.

O modelamento microestrutural da recristalização permite variar grandezas tais como: distribuição espacial de núcleos e forma funcional da velocidade de crescimento. Além disto, permite a quantificação exata de todos os parâmetros microestruturais relevantes tais como: fração volumétrica recristalizada, área de interfaces por unidade de volume, distribuição de tamanhos dos grãos dentre outros. Obter tais grandezas experimentalmente é um trabalho demorado que envolve erros experimentais consideráveis.

\section{MATERIAIS E MÉTODOS}

\subsection{Descrições da Simulação}

Foi simulada a reação sequencial de crescimento de duas fases distintas em uma mesma matriz. Foi utilizada a metodologia do Cone Causal, para o crescimento das fases. O sistema estudado era contido por uma matriz cúbica de $304^{3}$ células, e duas fases com as mesmas quantidades de núcleos iniciais e velocidades de crescimento iguais, $G_{1}=G_{2}$. O programa foi escrito em linguagem Fortran 2003, e compilado pelo Visual Studio $2012^{\circledR}$. A visualização das microestruturas transformadas foi realizada com auxílio do programa Tecplot $360^{\circledR}$ versão 2010, a elaboração dos gráficos foi realizada com o auxílio do programa Wolfram Mathematica $9^{\circledR}$. Na entrada de dados da simulação, foram escolhidos 64 núcleos para cada fase, totalizando 128 núcleos, o início da nucleação da reação 2 foi determinada para uma fração volumétrica transformada na matriz correspondente à

\footnotetext{
* Contribuição técnica ao $69^{\circ}$ Congresso Anual da ABM - Internacional e ao 14ํㅡㄹ ENEMET - Encontro Nacional de Estudantes de Engenharia Metalúrgica, de Materiais e de Minas, 21 a 25 de julho de 2014, São Paulo, SP, Brasil.
} 


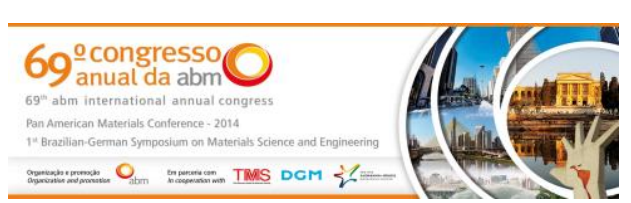

$30 \%$. Para simular uma amostra infinita foram utilizadas condições de contorno periódicas para todas as direções da matriz cúbica. O programa foi desenvolvido para apresentar como resultados de simulação: tempo de reação, fração volumétrica transformada, área superficial por unidade de volume, e velocidade de crescimento de cada fase. As grandezas podem ser obtidas no início meio e final da simulação, e são representadas em termos de reação total, e individualmente para cada fase.

\subsection{Reações Sequenciais}

Nesta seção, as expressões estão de acordo com o novo método analítico desenvolvido por Rios e Villa [9]. As expressões calculadas foram comparadas com os resultados da simulação.

Suponha que existam duas reações sequenciais denotadas como reação 1 e 2 . A reação 1 inicia-se no tempo $t$ e a reação 2 inicia-se no tempo $t_{2}$, sendo $t_{2}>t$. Os demais parâmetros foram considerados iguais para as duas reações. Logo as velocidades de crescimento são constantes, $G_{1}=G_{2}$. A nucleação é por saturação de sítios em ambos, e os núcleos estão localizados aleatoriamente no espaço, o número de núcleos por unidade de volume é $N_{V 1}=N_{V 2}$. A Fração volumétrica $\left(V_{V}\right)$ para ambas as fases podem ser estimados por:

$V_{V 1}(t)=1-\exp \left(-\frac{4 \pi}{3} N_{V 1} G_{1}^{3} t^{3}\right)$

Para $t>t_{2}$ :

$V_{V 2}(t)=1-\exp \left(-\frac{4 \pi}{3} N_{V 2} G_{2}^{3}\left(t-t_{2}\right)^{3}\right)$

Os valores experimentais de Fração volumétrica, $V^{*} v$ para cada fase são calculados de acordo com as Equações:

Para $\mathrm{t}<\mathrm{t} 2$ :

$V_{V 1}^{*}(t)=\int_{0}^{t} 4 \pi N_{V 1} G_{1}^{3} s^{2} \exp \left(-\frac{4 \pi}{3} N_{V 1} G_{1}^{3} s^{3}\right) d s$

$V_{V 2}^{*}(t)=0$

Para $t>t_{2}$ :

$V_{V 12}^{*}(t)=\int_{t}^{t_{2}} 4 \pi N_{V 1} G_{1}^{3} s^{2} \exp \left(-\frac{4 \pi}{3}\left(N_{V 1} G_{1}^{3} s^{3}+N_{V 2} G_{2}^{3}\left(s-s_{2}\right)^{3}\right)\right) d s$

$V_{V 22}^{*}(t)=\int_{t}^{t_{2}} 4 \pi N_{V 2} G_{2}^{3}\left(s-s_{2}\right)^{2} \exp \left(-\frac{4 \pi}{3}\left(N_{V 1} G_{1}^{3} s^{3}+N_{V 2} G_{2}^{3}\left(s-s_{2}\right)^{3}\right)\right) d s$

A Fração Volumétrica da reação total pode ser obtida através de:

Para $\mathrm{t}<\mathrm{t} 2$ :

$V_{V}^{*}(t)=V_{V 1}^{*}(t)+V_{V 2}^{*}(t)$

\footnotetext{
* Contribuição técnica ao 69ำ Congresso Anual da ABM - Internacional e ao 14ํㅡㄹ ENEMET - Encontro Nacional de Estudantes de Engenharia Metalúrgica, de Materiais e de Minas, 21 a 25 de julho de 2014, São Paulo, SP, Brasil.
} 
Para $t>t_{2}$ :

$V_{V}^{*}(t)=V_{V 12}^{*}(t)+V_{V 22}^{*}(t)$

A Quantidade de área interfacial experimental entre material transformado e não transformado por unidade de volume $S^{*}$, para cada fase, pode ser encontrada por:

Para $\mathrm{t}<\mathrm{t}_{2}$ :

$S_{V 1}^{*}(t)=4 \pi N_{V 1} G_{1}^{2} t^{2} \exp \left(-\frac{4 \pi}{3} N_{V 1} G_{1}^{3} t^{3}\right)$

$S_{V 2}^{*}(t)=0$

Para $t>t_{2}$ :

$$
\begin{aligned}
& S_{V 12}^{*}(t)=4 \pi N_{V 1} G_{1}^{2} t^{2} \exp \left(-\frac{4 \pi}{3}\left(N_{V 1} G_{1}^{3} t^{3}+N_{V 2} G_{2}^{3}\left(t-t_{2}\right)^{3}\right)\right) \\
& S_{V 22}^{*}(t)=4 \pi N_{V 2} G_{2}^{2}\left(t-t_{2}\right)^{2} \exp \left(-\frac{4 \pi}{3}\left(N_{V 1} G_{1}^{3} t^{3}+N_{V 2} G_{2}^{3}\left(t-t_{2}\right)^{3}\right)\right)
\end{aligned}
$$

As quantidades para a reação total são obtidas por:

Para $\mathrm{t}<\mathrm{t}_{2}$ :

$S_{V}^{*}(t)=S_{V 1}^{*}(t)+S_{V 2}^{*}(t)$

Para $t>t_{2}$ :

$S_{V}^{*}(t)=S_{V 12}^{*}(t)+S_{V 22}^{*}(t)$

Podemos estimar a velocidade G por:

$G(t, x)=\frac{1}{S_{V}(t, x)} \frac{\partial V_{V}(t, x)}{\partial t}$

O caminho microestrutural pode ser estimado realizando-se uma plotagem paramétrica entre as equações de $S_{V}$ e $V_{V}$, para essa operação vale ressaltar que, como demonstrado anteriormente, as equações de $S_{V}$ e $V_{V}$ de cada fase mudam com o tempo t.

\section{RESULTADOS E DISCUSSÃO}

Como resultados foram obtidos gráficos construídos com dados a partir da simulação pelo Cone Causal, que são representados por pontos e com dados previstos a partir das equações analíticas descritas a cima, que são representados por linhas sólidas. A fim de evitar sobrecarregá-los nem todos os pontos de simulação foram plotados. Com esses gráficos pode-se então comparar os métodos. Foram utilizados distribuição aleatória de núcleos iniciais tanto para a fase 1 quanto para a fase 2. A Figura 1 é a representação esquemática da microestrutura simulada e de sua evolução, nela pode-se evidenciar a nucleação da fase 2 em 30\% de fração volumétrica transformada na matriz.

* Contribuição técnica ao 69 Congresso Anual da ABM - Internacional e ao 14ํㅡㄹ ENEMET - Encontro Nacional de Estudantes de Engenharia Metalúrgica, de Materiais e de Minas, 21 a 25 de julho de 2014, São Paulo, SP, Brasil. 
Villa [9]. A evolução microestrutural de cada fase pôde ser bem representada pelo método analítico.

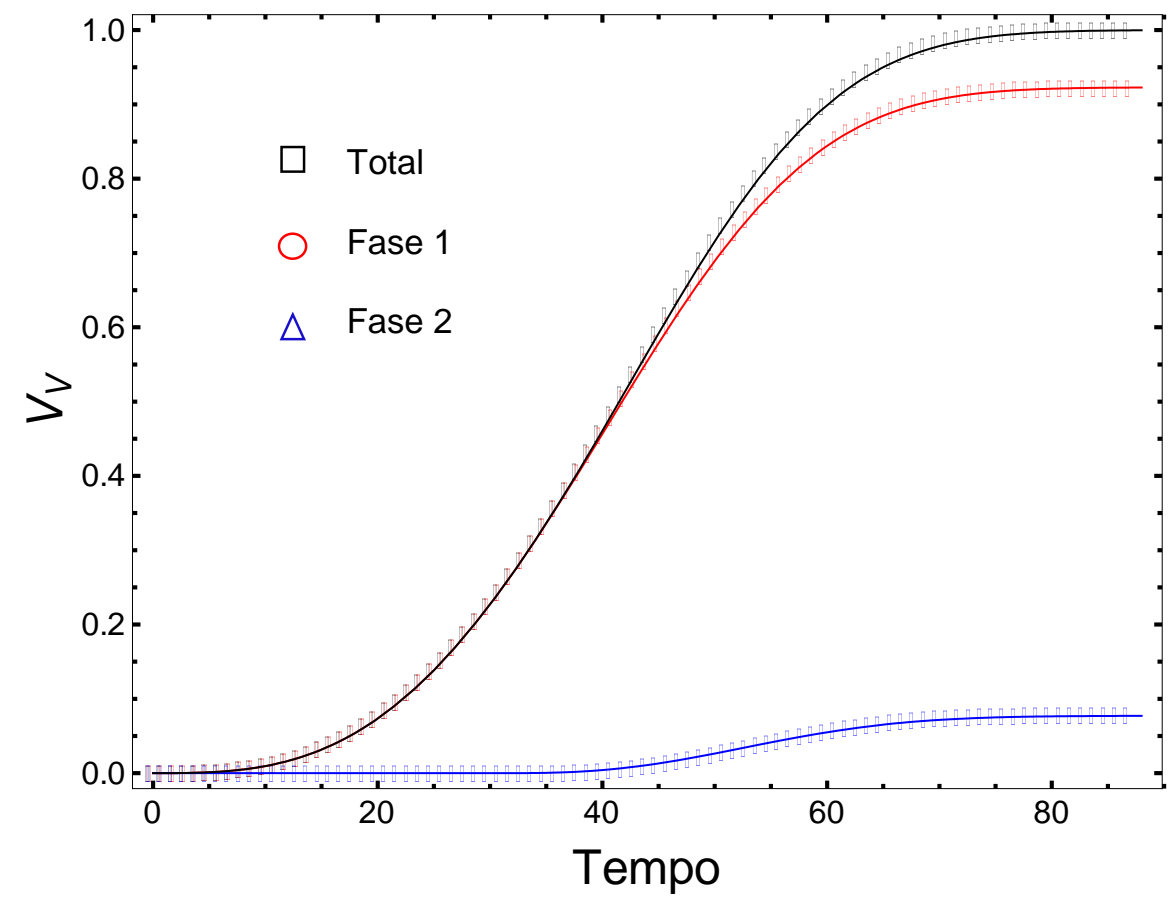

Figura 2: Cinética de evolução de duas fases crescendo sequencialmente versus tempo de transformação.

Assim como foi observado na fração volumétrica transformada em função do tempo de reação, na Figura 3 houve uma boa concordância, entre as curvas de $S_{V}$. simuladas e calculadas analiticamente.

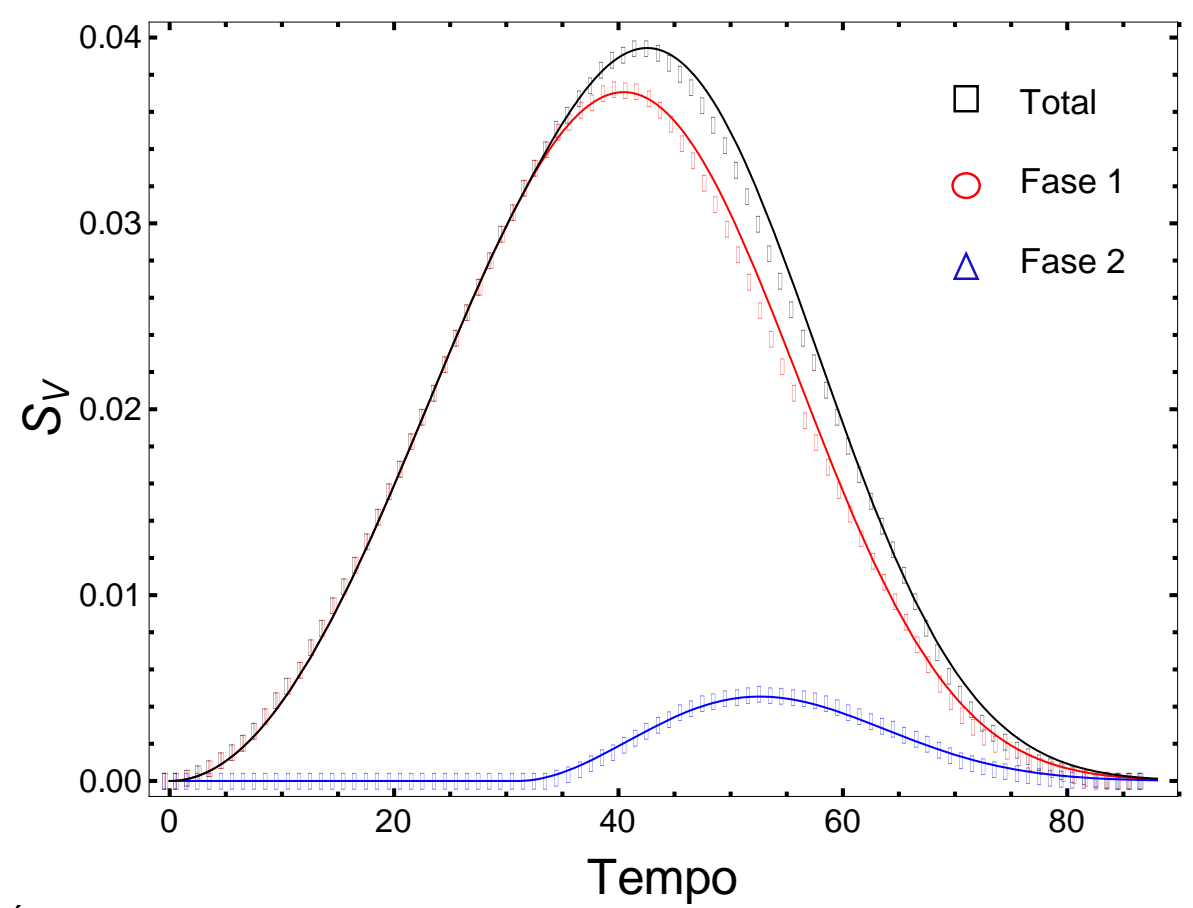

Figura 3: Área superficial por unidade de volume $S_{V}$ versus tempo de transformação de duas fases crescendo sequencialmente.

* Contribuição técnica ao $69^{\circ}$ Congresso Anual da ABM - Internacional e ao 14ํㅡㄹ ENEMET - Encontro Nacional de Estudantes de Engenharia Metalúrgica, de Materiais e de Minas, 21 a 25 de julho de 2014, São Paulo, SP, Brasil. 
$\mathrm{Na}$ Figura 4 têm-se uma boa concordância entre as velocidades simuladas e calculadas analiticamente, pode-se evidenciar que as fases possuem, em média, velocidades iguais. Nota-se que, a fase 2 tem uma maior dispersão, isso pode ser explicado, devido seu surgimento em um tempo posterior ao da fase 1, pois a mesma influencia negativamente no crescimento da fase 2.

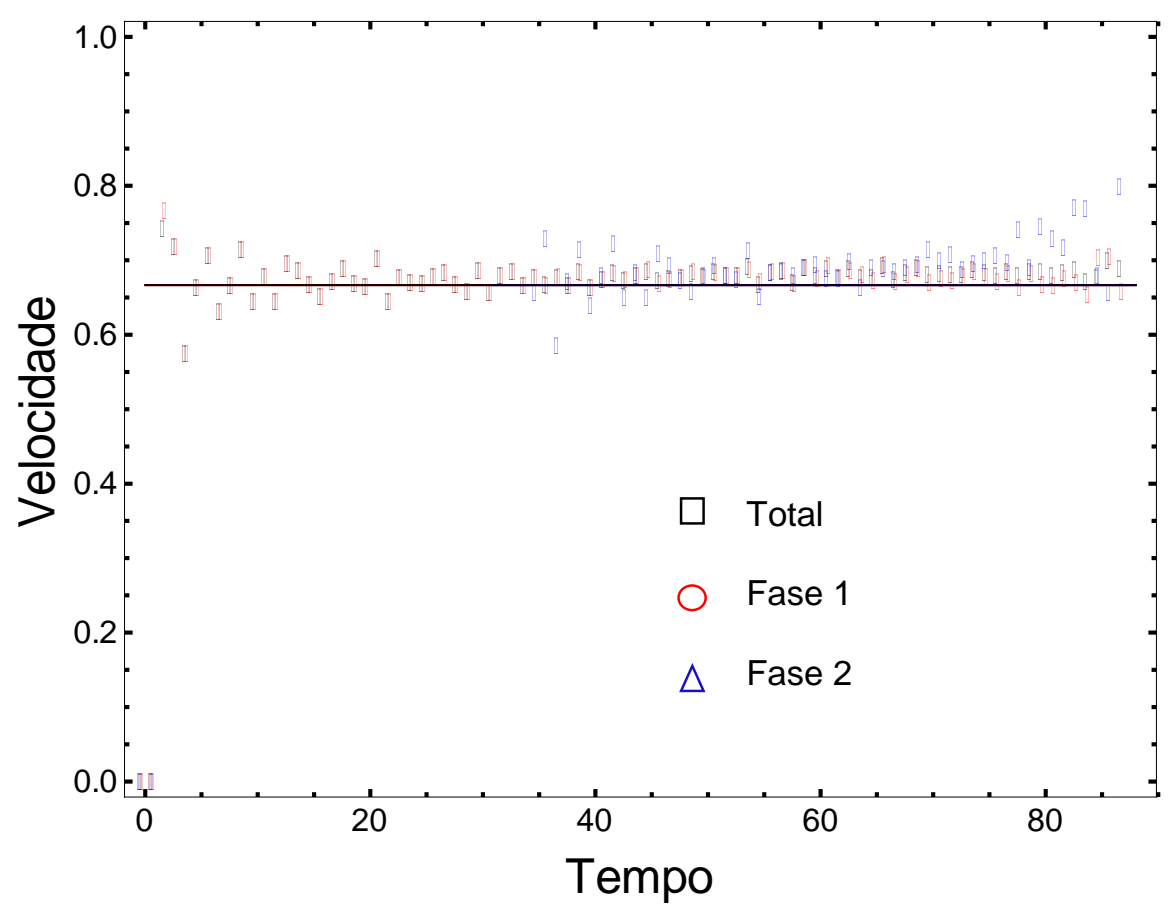

Figura 4: Velocidade de avanço das interfaces transformadas versus tempo de transformação de duas fases crescendo sequencialmente.

$\mathrm{Na}$ Figura 5 novamente observa-se que o caminho microestrutural apresenta uma boa concordância entre a simulação e a metodologia analítica.

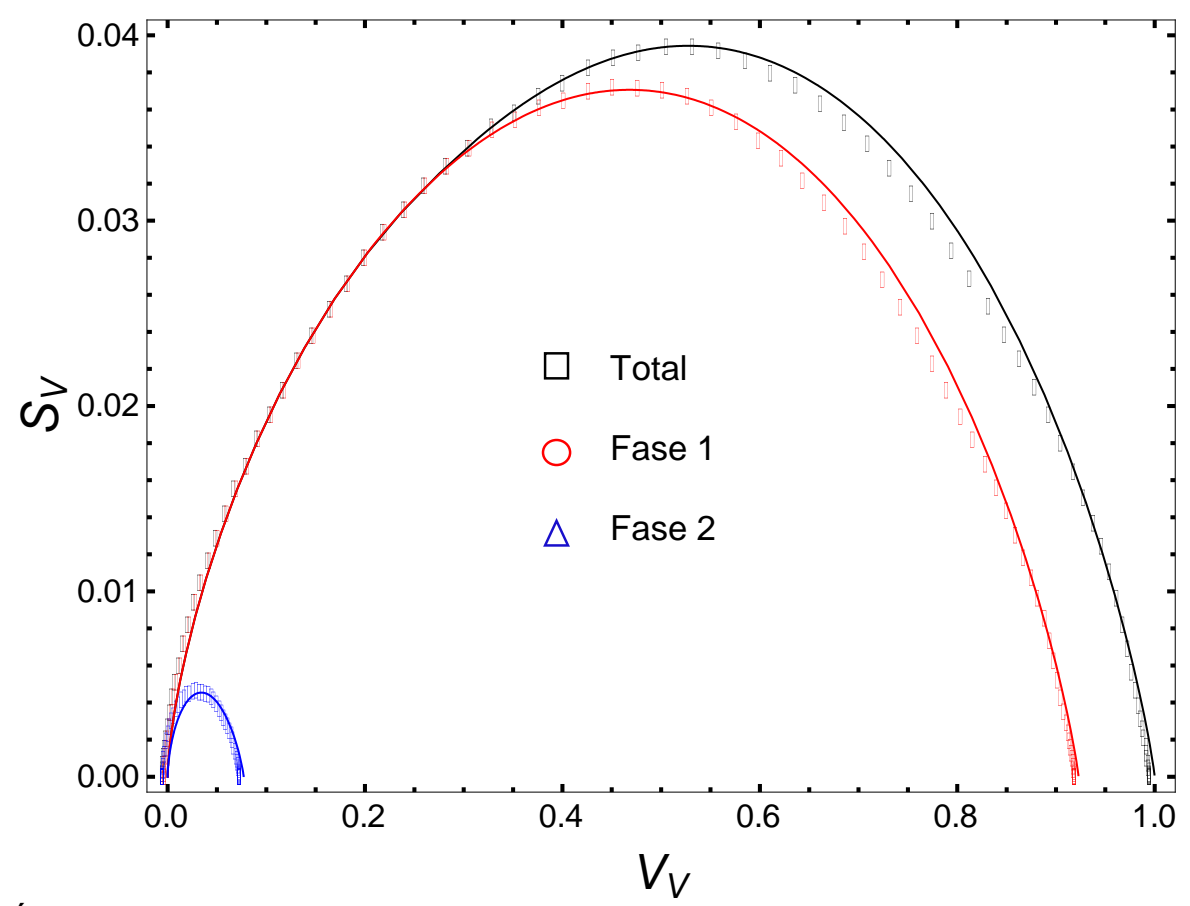

Figura 5: Área superficial por unidade de volume $S_{V}$ versus fração volumétrica $V_{V}$ de duas fases crescendo sequencialmente.

* Contribuição técnica ao 69 Congresso Anual da ABM - Internacional e ao 14ํㅡㄹ ENEMET - Encontro Nacional de Estudantes de Engenharia Metalúrgica, de Materiais e de Minas, 21 a 25 de julho de 2014, São Paulo, SP, Brasil. 


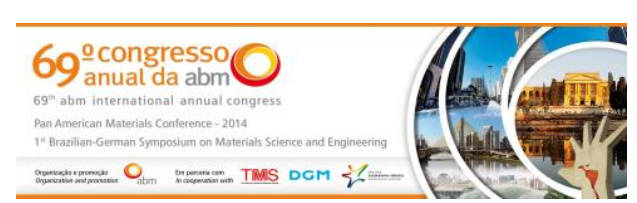

\section{CONCLUSÃO}

Neste trabalho o Cone Causal mostrou-se mais uma vez ser um método eficiente em simulação de transformações de fase. Foi possível simular com fidelidade os casos estudados analiticamente neste artigo. Todas as cinéticas de transformação apresentaram ótimas concordâncias com o modelo analiticamente exato de Rios e Villa [9]. É importante ressaltar que nenhum ajuste de curvas foi realizado nesse trabalho. Visualizando-se as curvas de caminho microestrutural pode-se concluir também que as formas geométricas foram mantidas ao longo da simulação.

A fase 1 tem certa influência sobre a fase 2. Este fato se dá devido ao surgimento posterior da fase 2, que é envolvida pela fase 1, o que também explica a baixa fração volumétrica da fase 2 em relação à fase 1 e uma maior variação da velocidade da fase 2 , como mencionado acima.

\section{Agradecimentos}

Agradecemos ao professor orientador Paulo Rangel Rios, e ao Doutor Weslley Luiz da Silva Assis por toda a assistência dada. A Universidade Federal Fluminense (UFF). Aos órgãos de Fomento: CNPQ, CAPES e FAPERJ.

\section{REFERÊNCIAS}

1 Vandermeer RA, Jensen DJ. Microstructural path and temperature dependence of recrystallization in commercial aluminium. Acta Materialia. 2001;39:2083-94.

2 Yamamoto T, Sakuma T. Application of Microstructural path analysis to abnormal grain growth of BaTiO3 with an excess TiO2. Scripta Materialia. 1998;39(12):1713-17.

3 Rios PR, Guimarães JRC. Microstructural path analysis of athermal martensite. Scripta Materialia. 2007;57:1105-08.

4 Price CW. Use of Kolmogorov-Johnson-Mehl-Avrami Kinetics in Recrystallization of Metals and Crystallization of Metallic Glasses. Acta Metallurgica and Materialia. 1990;38(5):727-738.

5 Johnson WA, Mehl RF. Mehl RF. Reaction kinetics in processes of nucleation and growth. Metallurgical Transactions Soc. AIME. 1939;135:416-441.

6 Kolmogorov AN. Izv. Akad. Nauk. USSR-Ser-Matemat. 1937;1(3):355.

7 Avrami M. Kinetics of phase change. I General theory. Journal of Chemical Physics. 1939;7:1103-12.

8 Avrami M. Kinetics of phase change. II. Journal of Chemical Physics. 1940;8:212-224.

9 Rios PR, Villa E. Transformation kinetics for inhomogeneous nucleation. Acta Mater. 2011;58:2752-68.

10 Rios PR, Villa E, Oliveira SC. New Methodology to Model Simultaneous and Sequential Reactions: Main Results And Applications. Materials Science Forum. 2012;706-709:149156.

11 Magnusson H, Jensen DJ, Hutchinson B. Scripta Mater. 2001;44:435.

\footnotetext{
* Contribuição técnica ao $69^{\circ}$ Congresso Anual da ABM - Internacional e ao 14ํㅡㄹ ENEMET - Encontro Nacional de Estudantes de Engenharia Metalúrgica, de Materiais e de Minas, 21 a 25 de julho de 2014, São Paulo, SP, Brasil.
} 